\title{
Subcellular microRNAs in diabetic cardiomyopathy
}

\author{
Huaping $\mathrm{Li}^{1,2}$, Jiahui Fan ${ }^{1,2}$, Chen Chen ${ }^{1,2}$, Dao Wen Wang ${ }^{1,2}$ \\ ${ }^{1}$ Division of Cardiology, Department of Internal Medicine, Tongji Hospital, Tongji Medical College, Huazhong University of Science and \\ Technology, Wuhan, China; ${ }^{2}$ Hubei Key Laboratory of Genetics and Molecular Mechanisms of Cardiological Disorders, Wuhan, China \\ Contributions: (I) Conception and design: H Li, C Chen, DW Wang; (II) Administrative support: DW Wang; (III) Provision of study materials or \\ patients: All authors; (IV) Collection and assembly of data: All authors; (V) Data analysis and interpretation: H Li; (VI) Manuscript writing: All \\ authors; (VII) Final approval of manuscript: All authors. \\ Correspondence to: Dao Wen Wang; Chen Chen. Division of Cardiology, Department of Internal Medicine, Tongji Hospital, Tongji Medical College, \\ Huazhong University of Science \& Technology, 1095\# Jiefang Ave., Wuhan 430030, China. Email: dwwang@tjh.tjmu.edu.cn; \\ chenchen@tjh.tjmu.edu.cn.
}

\begin{abstract}
Cardiovascular complications are the leading causes of diabetes-related morbidity and mortality. The high incidence and poor prognosis of heart failure in diabetic patients have been associated, in part, to the presence of an underlying cardiomyopathy characterized by cardiac hypertrophy, cardiomyocytes apoptosis, and fibrosis. It has been unclear about the mechanism that connects diabetes mellitus to the development of cardiovascular dysfunction. Micro(mi)RNAs represent a class of small, 18- to 28-nucleotidelong, non-coding RNA molecules. MiRNAs typically suppress gene expression at the post-transcriptional levels by binding directly to the 3'-UTR of the target mRNAs in the cytoplasm. Interestingly, recent studies suggest that miRNAs may also regulate gene expression in a positive manner. Our recent studies have shown that subcellular miRNAs, such as cytosol-, mitochondria- and nucleus-localized miRNAs, were dramatically dysregulated in diabetic cardiomyopathy. Specifically, cytoplasm localized miRNAs regulate genes expression in a post-transcriptional manner. Nuclear localized miRNAs regulate gene transcription or chromosomal reconstruction through the non-canonical mechanism. Mitochondrial miRNAs stimulate, rather than repress, the translation of specific mitochondrial genome-encoded transcripts. By reviewing these latest discovered functions of subcellular miRNAs in diabetic animal models, we identified new mechanistic insights for diabetic cardiomyopathy. Understanding the nature of subcellular miRNAs will provide new therapeutic targets against diabetes-associated cardiac complications in the near future.
\end{abstract}

Keywords: Subcellular miRNAs; diabetic cardiomyopathy; transcription; translation

Submitted Mar 05, 2020. Accepted for publication Sep 25, 2020.

doi: 10.21037/atm-20-2205

View this article at: http://dx.doi.org/10.21037/atm-20-2205

By 2025, the number of individuals with diabetes worldwide is predicted to reach $\sim 300$ million. Cardiovascular disease, which often leads to heart failure, accounts for more than $80 \%$ of mortality resulting from diabetes. Therefore, this study reviewed the mechanistic insights into diabetes-induced cardiac dysfunction in order to promote the development of treatment.

\section{Diabetes-induced cardiac dysfunction: vascular or cardiomyocyte disorder?}

Diabetes is a major risk factor for the development of heart failure (1). The Framingham Study reported approximate two- and five-fold increases in the risk of heart failure in diabetic men and women, respectively (2). The pathogenesis of heart failure in diabetes can be largely attributed to the cardiotoxic tetrad of coronary artery disease (CAD): hypertension, diabetic cardiomyopathy, and extracellular fluid volume expansion $(3,4)$. Among these cardiovascular complications, $\mathrm{CAD}$ is regarded as the leading cause of morbidity and mortality in patients with diabetes (5). Indeed, together with the primary prevention United Kingdom Prospective Diabetes Study (UKPDS), the Action 
to Control Cardiovascular Risk in Diabetes (ACCORD) trial, the Action in Diabetes and Vascular Disease (ADVANCE) trial, and the Veterans Affairs Diabetes Trial (VADT), all showed that individuals who were subjected to intensive, rather than standard glycemic, control had a statistically significant reduction in myocardial infarctionassociated morbidity (6). However, intensive glycemic control did not reduce the risk of hospital admission for heart failure, which is somewhat surprising given the importance of $\mathrm{CAD}$ in the pathogenesis of heart failure $(3,6)$.

Several studies have provided clues for the observation that an increase in the frequency of congestive heart failure in diabetic individuals is more likely be caused by cardiomyopathy than ischemic heart disease (7). Traditionally, diabetic patients have an increased incidence of heart failure, which has been attributed to coexisting ischemic or hypertensive heart disease (8). However, recent scientific evidence suggests that diabetic cardiomyopathy is being considered more as a distinct nosologic entity, independent of the co-existence of CAD, hypertension, or other risk factors $(8,9)$. Although coronary heart disease, hypertension and diabetic cardiomyopathy seem to mutually enhance the other's progression, possibly due to shared pathophysiological processes, such as reactive oxygen species (ROS) and inflammation (10-14), several differences have been noted between the two. Specifically, diabetic cardiomyopathy has been reported to show similar features of diastolic dysfunction (14), which is especially apparent in asymptomatic individuals as the earliest sign of heart failure. Most imaging evidence in patients with diabetes has not been found to show a significant decrease in ejection fraction/systolic dysfunction (15-17). The common histological characteristic of diabetic cardiomyopathy is the presence of interstitial and/or perivascular fibrosis. Diabetic cardiomyopathy can lead to global hypertrophy and apoptosis of cardiomyocytes (18), while ischemic heart disease usually induces cardiomyocyte apoptosis and fibrosis localized to the ischemic site (19). Diastolic dysfunction is one of the early manifestations in both diabetic cardiomyopathy and hypertensive heart disease (20). However, previous studies reported that hypertensive animals showed greater vascular changes but less myocardial damage than the more severely affected hypertensive-diabetic animals (21).

Currently, diabetic cardiomyopathy is typically defined as structural and functional abnormalities of the myocardium in diabetic patients without CAD or hypertension (22). However, myocardial abnormalities might also present in diabetic patients with CAD and/or hypertension; in such cases, assessing the specific contribution of diabetic cardiomyopathy to overall ventricular dysfunction is challenging (23). Clinically, it is unrealistic to diagnose diabetic cardiomyopathy only in the absence of CAD, hypertension, or any other form of cardiac disease. On this basis, researchers have recently proposed that diabetic cardiomyopathy might be defined as "cardiac abnormalities not wholly explained by other cardiovascular or noncardiovascular causes and likely to be due to diabetes" (24).

Diabetic cardiomyopathy most often occurs alongside other cardiovascular conditions, although it may also be the sole cause of cardiac disease (24). Therefore, in this review, we focus on the frequent, but often forgotten, occurrence of diabetic cardiomyopathy as a cardiovascular complication of diabetes characterized by cardiac hypertrophy and myocardial fibrosis (25).

\section{Mechanisms that contribute to the development of diabetic cardiomyopathy}

Several hypotheses have been proposed to explain the mechanisms underlying the development of diabetic cardiomyopathy.

\section{Lipotoxicity}

Increased myocardial free fatty acid (FFA) usage and reduced glucose oxidation have been observed in patients with type 1 and type 2 diabetes (26,27). Griffin et al. reported that glucose could directly activate the translation of fatty acid translocase (CD36) by regulating the upstream open reading frame in the 5 -untranslated region (UTR) of CD36 mRNA (28); consequently, an increased amount of FFA was imported into cardiomyocytes, even when the levels of circulating FFA were normal. Metabolism of high levels of FFA in cardiomyocytes requires increased oxygen consumption and leads to intracellular accumulation of toxic intermediates that may negatively influence myocardial performance through reduced availability of adenosine triphosphate (ATP) $(29,30)$.

\section{Mitochondrial dysfunction}

A study by Bugger and Abel revealed impaired respiratory capacity, mitochondrial oxidative stress, and abnormal mitochondrial ultrastructure in rodent models of insulindependent and non-insulin-dependent diabetes (31). 
Metabolic stress-induced mitochondrial dysfunction increases mitochondrial ROS generation and impairs oxidative phosphorylation resulting in cardiomyocyte hypertrophy and cell death.

\section{Impaired calcium $\left(\mathrm{Ca}^{2+}\right)$ bandling}

In their study, Jia et al. found that the hearts of type 1 and 2 diabetic mice had elevated intracellular resting $\mathrm{Ca}^{2+}$, prolonged intracellular $\mathrm{Ca}^{2+}$ decay, slowed $\mathrm{Ca}^{2+}$ transients, reduced sarcoplasmic reticulum $\mathrm{Ca}^{2+}$ pumping, and impaired sarcoplasmic reticulum $\mathrm{Ca}^{2+}$ reuptake (32). These observations suggest that impaired cardiomyocyte $\mathrm{Ca}^{2+}$ handling may play a key role in the development of cardiac diastolic dysfunction.

\section{Myocardial fibrosis and myocardial remolding}

Fibrosis, which can be either perivascular or interstitial, is the most typical cardiac-related finding in diabetic patients (33). Hyperglycemia induces abnormal gene expression and altered signal transduction, leading to cardiac hypertrophy and cardiomyocyte apoptosis. Hyperglycemia may also directly induce cardiomyocyte necrosis, resulting in increased collagen deposition, fibrosis, and cardiac remodeling in the heart (30). Dysregulation of extracellular matrix degradation due to the remodeling of matrix metalloproteinases (MMPs), in particular reduced expression of MMP-2, contributes to increased connective tissue content in diabetic hearts $(34,35)$.

\section{Signaling patbways}

A number of signaling pathways have been identified as important contributors to the development of diabetic cardiomyopathy, including the adenosine monophosphate protein kinase (AMPK), peroxisome proliferators-activated receptors (PPARs), O-linked beta-N-acetylglucosamine (O-GlcNAc), and protein kinase C (PKC) pathways (32). The AMPK pathway, for instance, is impaired in diabetic cardiomyopathy. AMPK enhances the expression of glucose transporter type 4 (GLUT4) and, subsequently, insulin-induced glucose uptake. Therefore, AMPK activation plays a protective role against the progression of diabetic cardiomyopathy (36). Peroxisome proliferatoractivated receptor $\gamma$ (PPAR- $\gamma$ ) has also shown cardiac antihypertrophic and anti-inflammatory effects, with PPAR- $\gamma$ agonists enhancing insulin sensitivity and improving glucose uptake in cardiomyocytes (37). Dassanayaka and Jones observed sustained O-GlcNAc signaling in the diabetic heart, which could exert detrimental effects on mitochondrial function and energy generation in cardiac function (38). PKC signaling pathways are activated in diabetic cardiomyopathy in response to hyperglycemia and insulin resistance. PKC $\alpha, \beta, \varepsilon, \theta$, and $\delta$ isoforms have been suggested to be involved in the development of diabetic cardiac hypertrophy (39). PKC $\beta 2$ has been shown to induce diastolic cardiac dysfunction in diabetic rats through caveolin-3 and insulin metabolic Akt/eNOS signaling (40). Furthermore, other signaling pathways, such as SGLT2, MAPK, NFkB, nuclear factor erythroid 2-related factor 2 (Nrf2), and cyclic adenosine 5'-monophosphate-responsive element modulator (CREM), have also been put forward as participators in diabetic cardiomyopathy (32).

\section{Other mechanisms}

Inflammation, autophagy, apoptosis/necrosis, the telomeretelomerase system, advanced glycation end-products, and endothelial dysfunction may also play roles in the development of diabetic cardiomyopathy $(41,42)$. Although these pathways are considered as separate mechanisms, they may interact with each other in complex ways. For instance, elevated FFA oxidation and lipotoxicity may promote mitochondrial dysfunction and oxidative stress; mitochondrial dysfunction and endoplasmic reticulum stress may increase apoptosis in cardiomyocytes; and oxidative stress, increased advanced glycation end-product signaling, and inflammation may promote apoptosis or the increased expression of pro-fibrotic genes (43). Overall, multiple potential mechanisms have been proposed and investigated for the development of diabetic cardiomyopathy. Novel mechanisms, such as miRNA and epigenetic mechanisms, remain relatively under-investigated and need to be further investigated (43). In the subsequent sections, we review the roles of various miRNAs, especially newly discovered subcellular localized miRNAs, in diabetic cardiomyopathy.

\section{MiRNAs and diabetic cardiomyopathy}

MiRNAs represent a class of small, 18- to 28-nucleotidelong, non-coding RNA molecules. MiRNAs typically suppress gene expression at the post-transcriptional level by binding directly to the 3'-UTR of target mRNAs (44). Interestingly, recent studies have suggested that miRNAs may also be positive regulators of gene expression $(45,46)$. 
Early studies reported the associations between altered miRNA expression and the pathologies of multiple diseases, including heart failure, atherosclerosis, diabetes, chronic kidney disease, and cancer (47-50). A recent study in mice revealed that dysregulation of 316 miRNAs was observed in diabetic hearts compared with controls. Among them, the expressions of 268 miRNAs remained significantly altered in diabetic mice, even after subsequent normoglycemia (51), indicating that miRNAs potentially contribute to the development of diabetic cardiomyopathy.

\section{Canonical mechanism}

For decades, miRNAs were reported as post-transcriptional regulators in the cytoplasm of multicellular organisms. MiRNAs typically exert their inhibitory effects by base pairing with the 3'-UTR of target mRNAs through their seed sequences $(52,53)$, which constitutes the canonical mechanism of miRNA function.

\section{Cytoplasmic miRNAs in diabetic cardiomyopathy}

Various animal studies have suggested that many cardiacenriched miRNAs are involved in the development of diabetic cardiomyopathy. MiR-133a, one of the predominantly expressed miRNAs in cardiac tissue, was found to be dramatically decreased in the hearts of streptozotocin (STZ)-induced type 1 diabetic animals. Chen et al. observed that miR-133a re-expression prevented a diabetes-induced increase in the expression of extracellular matrix protein and focal cardiac fibrosis, most likely by targeting transforming growth factor $\beta 1$ mRNA (54). In the hearts of mice with high-fat dietinduced type 2 diabetes, the levels of miR-451 were found to be significantly increased, while cardiomyocyte-specific miR-451 knockout mice showed ameliorated cardiac hypertrophy and contractile dysfunction. Calcium Binding Protein 39 (Cab39) was identified by Kuwabara et al. as a direct target of miR-451 in the heart (55). MiR-195 expression was increased in STZ-induced type 1 and $\mathrm{db} / \mathrm{db}$ type 2 diabetic mouse hearts. Knocking down of miR-195 expression in the heart was shown to significantly attenuate myocardial hypertrophy and improve myocardial function in STZ-treated mice via direct targeting of Sirt1 and Bcl-2 mRNA (56). Meanwhile, upregulation of miR-195 sufficiently induced apoptosis in cardiomyocytes and promoted cardiac dysfunction. MiR-155 was enhanced in the macrophages and the hearts of ovariectomyinduced diabetic mice. The delivery of gold nanoparticlebased miR-155 antagonist macrophages restored cardiac function in these mice (57). Upregulation of miR195 sufficiently induced apoptosis in cardiomyocytes and promoted cardiac dysfunction in diabetic hearts (56). MiR-34a overexpression impaired autophagy in high-glucose-induced cardiomyocytes in the hearts of diabetic mice; however, the downregulation of miR$34 \mathrm{a}$ restored autophagy, and thus ameliorated diabetic cardiomyopathy (58). In cardiomyocytes, miR-34a induced telomere shortening by reducing the level of protein phosphatase 1 nuclear targeting subunit, whereas the inhibition of miR-34a significantly enhanced telomere length and telomerase activity $(59,60)$. Interestingly, miR34 a inhibition also reduced high glucose-induced apoptosis in cardiomyocytes.

Our group systematically investigated the roles of miRNAs in diabetic cardiomyopathy. For example, miR$30 \mathrm{c}$ was significantly decreased in $\mathrm{db} / \mathrm{db}$ type 2 diabetic model mice. MiR-30c overexpression by a recombinant adeno-associated virus type 9 (rAAV9) vector reversed cardiac functional and structural changes in diabetic mice $(61,62)$. Mechanistically, miR-30c repressed beclin-1 expression by directly binding to its 3 '-UTR. MiR-30c overexpression inhibited the induction of beclin-1 and subsequent autophagy in diabetic hearts (61). Moreover, miR-30c also directly targeted and repressed the expression of PGC- $1 \beta$, which reduced the transcriptional activity of PPAR- $\alpha$. Glucose-fatty acid use, oxidative stress, lipid accumulation, ATP production abnormalities, and apoptosis mediated by PPAR- $\alpha$ were eliminated by treatment with miR-30c (61). Another miRNA enriched in the heart, miR21 , was specifically down-regulated in cardiomyocytes but remained unchanged in non-cardiomyocytes of $\mathrm{db} / \mathrm{db}$ and high-fat diet-induced type 2 diabetic mice. Remarkably, treatment with miR-21 effectively protected against the early impairment in cardiac diastolic dysfunction in $\mathrm{db} /$ $\mathrm{db}$ mice, which was shown by decreased ROS production, increased bioavailable nitric oxide, and improved diabetes-induced cardiomyocyte hypertrophy. Through bioinformatics analysis and Ago2 co-immunoprecipitation, we identified gelsolin, a member of the actin-binding protein family, which acted as a transcriptional cofactor in signal transduction, as a direct target of miR-21 (63). These findings suggest that changes to miRNAs localized in the cytoplasm contribute to the development of diabetic 


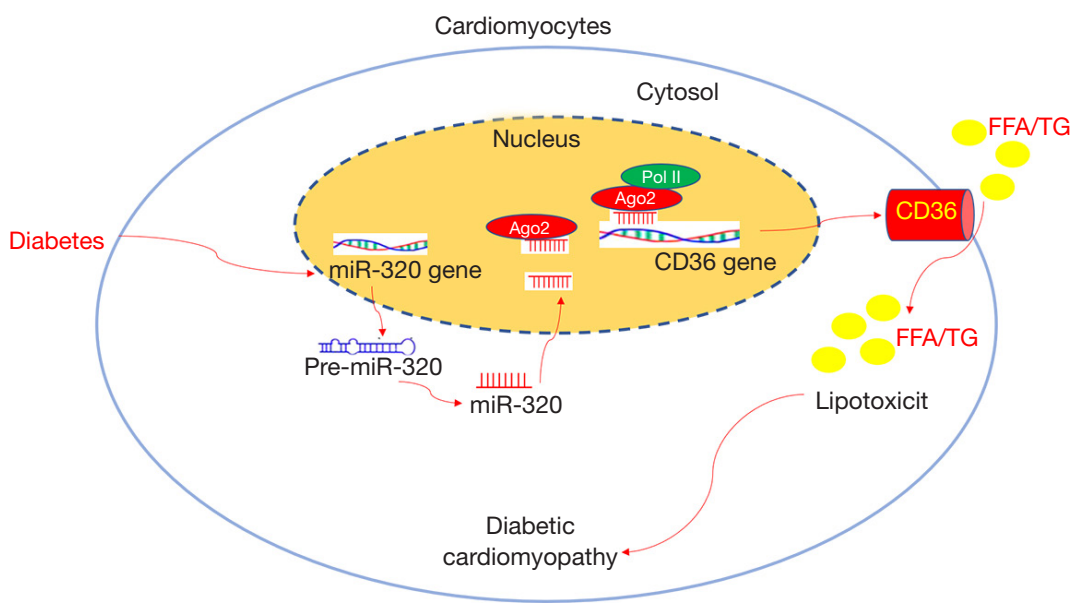

Figure 1 Nuclear miR-320 mediates diabetes-induced cardiac dysfunction by activating the transcription of the fatty acid metabolic gene CD36 to cause lipotoxicity in the heart. MiR-320, MicroRNA-320; FFA, free fatty acids; TG, triacylglycerol; CD36, cluster of differentiation 36; Ago2, Argonaute RISC Catalytic Component 2; Pol II, RNA polymerase II.

cardiomyopathy.

\section{Non-canonical mechanism}

Some miRNAs might localize in the nucleus or the mitochondria, as well as in the cytoplasm $(46,64)$. Stavast and Erkeland obtained strong evidence that nuclear miRNAs regulate the transcription of target genes by binding to reverse complementary sequences in promoter regions in their functional in vitro experiments using mammalian cells (65). Instead of negatively regulating gene transcription by binding to the 3'-UTR of target mRNAs, miRNAs localized in the mitochondria stimulate, rather than repress, the translation of specific mitochondrial genome-encoded transcripts. These subcellular localized miRNAs usually function through non-canonical mechanisms.

\section{Nuclear miRNAs in diabetic cardiomyopathy}

Most miRNAs can be found in both the nucleus and the cytoplasm, with some showing selective nuclear enrichment (66). For instance, in HeLa cells, miR-29b is mainly nuclear, whereas miR-29a is predominantly cytoplasmic (67). However, in comparison to many studies examining cytoplasm-localized miRNAs, the pathophysiological roles of nuclear miRNAs remain largely under-investigated. Our recent study demonstrated that a specific miRNA, miR-320, was significantly up-regulated in the diabetic myocardium of mice and human patients, and translocated into the nucleus to directly enhance CD36 expression (68).

MiR-320 acts as a small activating RNA in the nucleus at the transcriptional level. CD36 is a key target gene of miR-320, and the induced expression of CD36 is responsible for increased FFA uptake and cardiac lipid accumulation, thereby causing cardiac lipotoxicity and increased cardiomyocyte apoptosis. The delivery of rAAV9mediated miR-320 TUD (Tough Decoy) was shown to restore cardiac dysfunction in diabetic mice. These findings indicate that the miR-320/CD36 pathway links glucose toxicity to lipotoxicity in the heart (Figure 1). This study offered the first in vivo example of transcriptional activation by a natural small activating RNA and uncovered a novel mechanism for cardiac dysfunction induced directly by diabetes. Furthermore, this finding suggests a potential strategy for developing miRNA-based therapy for diabetesassociated cardiovascular complications (68).

\section{Mitochondrial miRNAs in diabetic cardiomyopathy}

Recently, miRNAs were reported to be present in the mitochondria. As early as 2009, several groups reported the detection of nuclear-coded miRNAs in rat liver mitochondria and HeLa cells (69-71). Later, Das et al. reported that miR-181c in rat mitochondria was involved in electron chain complex IV remodeling in 
cardiomyocytes (72). Another study of interest showed the redistribution of mitochondrial miRNAs in diabetic hearts. In STZ-induced type 1 diabetic hearts, Jagannathan et al.'s miRNA array analysis indicated that 78 miRNAs were differentially expressed in the mitochondrial subpopulation relative to the controls (73). However, the significance and functional consequences of these changes were not characterized. Our recent study demonstrated that 14 miRNAs were downregulated in the mitochondria of $\mathrm{db} / \mathrm{db}$ type 2 diabetic mouse hearts (74). Of these miRNAs, miR$92 a-2-5 p$ and let-7b-5p targeted mitochondrially encoded cytochrome B (mt-Cytb) and positively modulated $\mathrm{mt}-\mathrm{Cytb}$ expression. Overexpression of miR-92a-2-5p or let-7b$5 \mathrm{p}$ was also observed to decrease apoptosis and the level of mitochondrial ROS through the upregulation of mt-Cytb. Interestingly, rAAV9-mediated delivery of miR-92a-2-5p, but not let-7b-5p, could rescue cardiac diastolic dysfunction in the hearts of $d b / d b$ mice. Let-7b-5p not only upregulated mt-Cytb, but also downregulated insulin receptor substrate 1 in cytosol, resulting in a failure to improve diastolic dysfunction in $\mathrm{db} / \mathrm{db}$ mice (74). Our study gave the first in vivo example of mitochondria-localized miRNAs in diabetic cardiomyopathy and revealed a complicated pattern of miRNA regulation in the subcellular organelles during the disease.

\section{Conclusions and perspectives}

MiRNAs are commonly believed to serve as fine-tuning tools that play regulatory roles in virtually all diabetic cardiomyopathy mechanisms. Especially in type 1 and 2 diabetic hearts, an accumulation of lipids is frequently observed, which negatively influences myocardial performance through the excessive production of toxic intermediates. However, the mechanism by which glucose toxicity leads to lipotoxicity has not been well defined. Our recent study reveals that nuclear miR-320 might be one of the "missing links" between glucose toxicity and lipotoxicity in diabetic hearts. MiRNAs might also be associated with the signaling pathways involved in diabetic cardiomyopathy such as inflammation, autophagy, and apoptosis/necrosis. MiRNAs can act as the nodes of signaling networks that regulate the progression of diabetic cardiomyopathy. Signaling pathways are prime candidates for miRNAmediated regulation, and signaling complexes are the ideal targets for the degree of quantitative fluctuations imposed by miRNAs. Instead of focusing on protein-coded signaling pathways, which are difficult to target therapeutically, one could focus on their target miRNAs. However, facing the potential complexity of the miRNA-signaling network, future studies are needed to reveal the complicated crosstalk between miRNAs and diabetic cardiomyopathy-related signaling pathways using unambiguous and pathway-specific readouts in cultured cell or other model systems.

Until recently, little was known about the subcellular localization, functions, turnover, and dynamics of miRNAs. In fact, most miRNAs are present in both the nucleus and the cytoplasm. However, their pathophysiological functions and mechanisms are largely undetermined. Furthermore, miRNAs are also localized in other subcellular compartments. For instance, mitochondrial miRNAs that control the translation of mitochondrial genes could provide new mechanisms for mitochondrial dysfunction in diabetic cardiomyopathy.

Conclusively, miRNAs localized in the cytoplasm usually regulate gene expression through a post-transcriptional manner, which fell into the canonical mechanism of miRNAs. Nuclear miRNAs regulate gene transcription or chromosomal reconstruction through a non-canonical mechanism $(68,75)$. Mitochondrial miRNAs stimulate, rather than repress, the translation of specific mitochondrial genome-encoded transcripts by targeting their coding sequences, instead of the 3'-UTRs of target mRNAs, which represents another noncanonical mechanism of miRNAs (46).

A major drawback current studies on miRNAs is the absence of a strategy to investigate their cytosolic, mitochondrial, or nuclear effects independently. Thus, more sophisticated experimental systems must be developed to resolve the roles and functions of miRNAs in subcellular compartments (76). It is still unclear how subcellularly localized miRNAs work together to function in diabetic cardiomyopathy. Moreover, although many potential mechanisms have been proposed for diabetic cardiomyopathy, including lipotoxicity, ROS induction, and mitochondrial dysfunction, their effects are rather unclear, and questions remain regarding the factors involved in the initiation and progression of diabetic cardiomyopathy. A deeper understanding of the nature of subcellular miRNAs is needed to reveal new mechanisms and to identify potential novel therapeutic targets for diabetes-associated cardiac complications.

\section{Acknowledgments}

We thank our colleagues in Dr. Wang's group for stimulating discussions during the course of this 
investigation.

Funding: This work was supported by grants from the National Natural Science Foundation of China (nos. 81822002, 81630010, 91839302, 31771264, 81790624, and 31800973). The funders had no role in the study designs, data collection and analysis, manuscript preparation, or decision to publish.

\section{Footnote}

Peer Review File: Available at http://dx.doi.org/10.21037/ atm-20-2205

Conflicts of Interest: All authors have completed the ICMJE uniform disclosure form (available at http://dx.doi. org/10.21037/atm-20-2205). CC and DWW report grants from National Natural Science Foundation of China (NSFC) during the conduct of the study. The other authors have no conflicts of interest to declare.

Ethical Statement: The authors are accountable for all aspects of the work in ensuring that questions related to the accuracy or integrity of any part of the work are appropriately investigated and resolved.

Open Access Statement: This is an Open Access article distributed in accordance with the Creative Commons Attribution-NonCommercial-NoDerivs 4.0 International License (CC BY-NC-ND 4.0), which permits the noncommercial replication and distribution of the article with the strict proviso that no changes or edits are made and the original work is properly cited (including links to both the formal publication through the relevant DOI and the license). See: https://creativecommons.org/licenses/by-nc-nd/4.0/.

\section{References}

1. Schocken DD, Benjamin EJ, Fonarow GC, et al. Prevention of heart failure: a scientific statement from the American Heart Association Councils on Epidemiology and Prevention, Clinical Cardiology, Cardiovascular Nursing, and High Blood Pressure Research; Quality of Care and Outcomes Research Interdisciplinary Working Group; and Functional Genomics and Translational Biology Interdisciplinary Working Group. Circulation 2008;117:2544-65.

2. Kannel WB, Hjortland M, Castelli WP. Role of diabetes in congestive heart failure: the Framingham study. Am J
Cardiol 1974;34:29-34.

3. Gilbert RE, Krum H. Heart failure in diabetes: effects of anti-hyperglycaemic drug therapy. Lancet 2015;385:2107-17.

4. Bell DSH, Goncalves E. Heart failure in the patient with diabetes: Epidemiology, aetiology, prognosis, therapy and the effect of glucose-lowering medications. Diabetes Obes Metab 2019;21:1277-90.

5. Chopra S, Peter S. Screening for coronary artery disease in patients with type 2 diabetes mellitus: An evidence-based review. Indian J Endocrinol Metab 2012;16:94-101.

6. Turnbull FM, Abraira C, Anderson RJ, et al. Intensive glucose control and macrovascular outcomes in type 2 diabetes. Diabetologia 2009;52:2288-98.

7. Bell DS. Diabetic cardiomyopathy. A unique entity or a complication of coronary artery disease? Diabetes Care 1995;18:708-14.

8. Mytas DZ, Stougiannos PN, Zairis MN, et al. Diabetic myocardial disease: pathophysiology, early diagnosis and therapeutic options. J Diabetes Complications 2009;23:273-82.

9. Adeghate E, Singh J. Structural changes in the myocardium during diabetes-induced cardiomyopathy. Heart Fail Rev 2014;19:15-23.

10. Arauz-Pacheco C, Parrott MA, Raskin P; American Diabetes Association. Treatment of hypertension in adults with diabetes. Diabetes Care 2003;26 Suppl 1:S80-2.

11. Su W, Guo Z, Randall DC, et al. Hypertension and disrupted blood pressure circadian rhythm in type 2 diabetic db/db mice. Am J Physiol Heart Circ Physiol 2008;295:H1634-41.

12. Cheung BM, Li C. Diabetes and hypertension: is there a common metabolic pathway? Curr Atheroscler Rep 2012;14:160-6.

13. Escobar E. Hypertension and coronary heart disease. J Hum Hypertens 2002;16 Suppl 1:S61-3.

14. Wang Y, Yu Q, Fan D, et al. Coronary heart disease in type 2 diabetes: mechanisms and comprehensive prevention strategies. Expert Rev Cardiovasc Ther 2012;10:1051-60.

15. Skali H, Shah A, Gupta DK, et al. Cardiac structure and function across the glycemic spectrum in elderly men and women free of prevalent heart disease: the Atherosclerosis Risk In the Community study. Circ Heart Fail 2015;8:448-54.

16. $\mathrm{Ng}$ AC, Delgado V, Bertini M, et al. Findings from left ventricular strain and strain rate imaging in asymptomatic patients with type 2 diabetes mellitus. Am J Cardiol 2009;104:1398-401. 
17. Garagan SF. The differential diagnosis of diabetic cardiopathy and ischemic heart disease in diabetics. Vrach Delo 1990;(2):61-3.

18. Crisafulli A, Pagliaro P, Roberto S, et al. Diabetic Cardiomyopathy and Ischemic Heart Disease: Prevention and Therapy by Exercise and Conditioning. Int J Mol Sci 2020;21:2896.

19. Gyongyosi M, Winkler J, Ramos I, et al. Myocardial fibrosis: biomedical research from bench to bedside. Eur J Heart Fail 2017;19:177-91.

20. Chacko BR, Karur GR, Connelly KA, et al. Left ventricular structure and diastolic function by cardiac magnetic resonance imaging in hypertrophic cardiomyopathy. Indian Heart J 2018;70:75-81.

21. Factor SM, Bhan R, Minase T, et al. Hypertensive-diabetic cardiomyopathy in the rat: an experimental model of human disease. Am J Pathol 1981;102:219-28.

22. Aneja A, Tang WH, Bansilal S, et al. Diabetic cardiomyopathy: insights into pathogenesis, diagnostic challenges, and therapeutic options. Am J Med 2008;121:748-57.

23. Miki T, Yuda S, Kouzu H, et al. Diabetic cardiomyopathy: pathophysiology and clinical features. Heart Fail Rev 2013;18:149-66.

24. Lee MMY, McMurray JJV, Lorenzo-Almoros A, et al. Diabetic cardiomyopathy. Heart 2019;105:337-45.

25. Bell DS. Heart failure: the frequent, forgotten, and often fatal complication of diabetes. Diabetes Care 2003;26:2433-41.

26. Herrero P, Peterson LR, McGill JB, et al. Increased myocardial fatty acid metabolism in patients with type 1 diabetes mellitus. J Am Coll Cardiol 2006;47:598-604.

27. Monti LD, Landoni C, Setola E, et al. Myocardial insulin resistance associated with chronic hypertriglyceridemia and increased FFA levels in Type 2 diabetic patients. Am J Physiol Heart Circ Physiol 2004;287:H1225-31.

28. Griffin E, Re A, Hamel N, et al. A link between diabetes and atherosclerosis: Glucose regulates expression of CD36 at the level of translation. Nat Med 2001;7:840-6.

29. Rodrigues B, Cam MC, McNeill JH. Metabolic disturbances in diabetic cardiomyopathy. Mol Cell Biochem 1998;180:53-7.

30. Kasznicki J, Drzewoski J. Heart failure in the diabetic population - pathophysiology, diagnosis and management. Arch Med Sci 2014;10:546-56.

31. Bugger H, Abel ED. Rodent models of diabetic cardiomyopathy. Dis Model Mech 2009;2:454-66.
32. Jia G, Hill MA, Sowers JR. Diabetic Cardiomyopathy: An Update of Mechanisms Contributing to This Clinical Entity. Circ Res 2018;122:624-38.

33. Fang ZY, Prins JB, Marwick TH. Diabetic cardiomyopathy: evidence, mechanisms, and therapeutic implications. Endocr Rev 2004;25:543-67.

34. Westermann D, Rutschow S, Jager S, et al. Contributions of inflammation and cardiac matrix metalloproteinase activity to cardiac failure in diabetic cardiomyopathy: the role of angiotensin type 1 receptor antagonism. Diabetes 2007;56:641-6.

35. Van Linthout S, Seeland U, Riad A, et al. Reduced MMP-2 activity contributes to cardiac fibrosis in experimental diabetic cardiomyopathy. Basic Res Cardiol 2008;103:319-27.

36. Zou MH, Xie Z. Regulation of interplay between autophagy and apoptosis in the diabetic heart: new role of AMPK. Autophagy 2013;9:624-5.

37. Jia G, DeMarco VG, Sowers JR. Insulin resistance and hyperinsulinaemia in diabetic cardiomyopathy. Nat Rev Endocrinol 2016;12:144-53.

38. Dassanayaka S, Jones SP. O-GlcNAc and the cardiovascular system. Pharmacol Ther 2014;142:62-71.

39. Li Z, Abdullah CS, Jin ZQ. Inhibition of PKC-theta preserves cardiac function and reduces fibrosis in streptozotocin-induced diabetic cardiomyopathy. Br J Pharmacol 2014;171:2913-24.

40. Lei S, Li H, Xu J, et al. Hyperglycemia-induced protein kinase $\mathrm{C}$ beta 2 activation induces diastolic cardiac dysfunction in diabetic rats by impairing caveolin-3 expression and Akt/eNOS signaling. Diabetes 2013;62:2318-28.

41. Srinivasan S, Hatley ME, Bolick DT, et al. Hyperglycaemia-induced superoxide production decreases eNOS expression via AP-1 activation in aortic endothelial cells. Diabetologia 2004;47:1727-34.

42. Al-Nimer MS, Al-Ani FS, Ali FS. Role of nitrosative and oxidative stress in neuropathy in patients with type 2 diabetes mellitus. J Neurosci Rural Pract 2012;3:41-4.

43. Bugger H, Abel ED. Molecular mechanisms of diabetic cardiomyopathy. Diabetologia 2014;57:660-71.

44. Bartel DP. MicroRNAs: target recognition and regulatory functions. Cell 2009;136:215-33.

45. Vasudevan S, Steitz JA. AU-rich-element-mediated upregulation of translation by FXR1 and Argonaute 2. Cell 2007;128:1105-18.

46. Zhang X, Zuo X, Yang B, et al. MicroRNA directly enhances mitochondrial translation during muscle 
differentiation. Cell 2014;158:607-19.

47. He M, Wang J, Yin Z, et al. MiR-320a induces diabetic nephropathy via inhibiting MafB. Aging (Albany NY) 2019;11:3055-79.

48. Fan J, Li H, Nie X, et al. MiR-665 aggravates heart failure via suppressing CD34-mediated coronary microvessel angiogenesis. Aging (Albany NY) 2018;10:2459-79.

49. Li H, Zhang X, Wang F, et al. MicroRNA-21 Lowers Blood Pressure in Spontaneous Hypertensive Rats by Upregulating Mitochondrial Translation. Circulation 2016;134:734-51.

50. Chen C, Wang Y, Yang S, et al. MiR-320a contributes to atherogenesis by augmenting multiple risk factors and down-regulating SRF. J Cell Mol Med 2015;19:970-85.

51. Costantino S, Paneni F, Luscher TF, et al. MicroRNA profiling unveils hyperglycaemic memory in the diabetic heart. Eur Heart J 2016;37:572-6.

52. Saliminejad K, Khorram Khorshid HR, Soleymani Fard $\mathrm{S}$, et al. An overview of microRNAs: Biology, functions, therapeutics, and analysis methods. J Cell Physiol 2019;234:5451-65.

53. Dragomir MP, Knutsen E, Calin GA. SnapShot: Unconventional miRNA Functions. Cell 2018;174:10381038.e1.

54. Chen S, Puthanveetil P, Feng B, et al. Cardiac miR-133a overexpression prevents early cardiac fibrosis in diabetes. J Cell Mol Med 2014;18:415-21.

55. Kuwabara Y, Horie T, Baba O, et al. MicroRNA-451 exacerbates lipotoxicity in cardiac myocytes and highfat diet-induced cardiac hypertrophy in mice through suppression of the LKB1/AMPK pathway. Circ Res 2015;116:279-88.

56. Zheng D, Ma J, Yu Y, et al. Silencing of miR-195 reduces diabetic cardiomyopathy in C57BL/6 mice. Diabetologia 2015;58:1949-58.

57. Jia C, Chen H, Wei M, et al. Gold nanoparticle-based miR155 antagonist macrophage delivery restores the cardiac function in ovariectomized diabetic mouse model. Int J Nanomedicine 2017;12:4963-79.

58. Ni T, Lin N, Lu W, et al. Dihydromyricetin Prevents Diabetic Cardiomyopathy via miR-34a Suppression by Activating Autophagy. Cardiovasc Drugs Ther 2020;34:291-301.

59. Boon RA, Iekushi K, Lechner S, et al. MicroRNA34 a regulates cardiac ageing and function. Nature 2013;495:107-10.

60. Marques FZ, Booth SA, Prestes PR, et al. Telomere dynamics during aging in polygenic left ventricular hypertrophy. Physiol Genomics 2016;48:42-9.

61. Chen C, Yang S, Li H, et al. Mir30c Is Involved in Diabetic Cardiomyopathy through Regulation of Cardiac Autophagy via BECN1. Mol Ther Nucleic Acids 2017;7:127-39.

62. Yin Z, Zhao Y, He M, et al. MiR-30c/PGC-1beta protects against diabetic cardiomyopathy via PPARalpha. Cardiovasc Diabetol 2019;18:7.

63. Dai B, Li H, Fan J, et al. MiR-21 protected against diabetic cardiomyopathy induced diastolic dysfunction by targeting gelsolin. Cardiovasc Diabetol 2018;17:123.

64. Meister G, Landthaler M, Patkaniowska A, et al. Human Argonaute2 mediates RNA cleavage targeted by miRNAs and siRNAs. Mol Cell 2004;15:185-97.

65. Stavast CJ, Erkeland SJ. The Non-Canonical Aspects of MicroRNAs: Many Roads to Gene Regulation. Cells 2019;8:1465.

66. Khudayberdiev SA, Zampa F, Rajman M, et al. A comprehensive characterization of the nuclear microRNA repertoire of post-mitotic neurons. Front Mol Neurosci $2013 ; 6: 43$.

67. Hwang HW, Wentzel EA, Mendell JT. A hexanucleotide element directs microRNA nuclear import. Science 2007;315:97-100.

68. Li H, Fan J, Zhao Y, et al. Nuclear miR-320 Mediates Diabetes-Induced Cardiac Dysfunction by Activating Transcription of Fatty Acid Metabolic Genes to Cause Lipotoxicity in the Heart. Circ Res 2019;125:1106-20.

69. Kren BT, Wong PY, Sarver A, et al. MicroRNAs identified in highly purified liver-derived mitochondria may play a role in apoptosis. RNA Biol 2009;6:65-72.

70. Barrey E, Saint-Auret G, Bonnamy B, et al. Pre-microRNA and mature microRNA in human mitochondria. PloS One 2011;6:e20220.

71. Bandiera S, Ruberg S, Girard M, et al. Nuclear outsourcing of RNA interference components to human mitochondria. PLoS One 2011;6:e20746.

72. Das S, Ferlito M, Kent OA, et al. Nuclear miRNA regulates the mitochondrial genome in the heart. Circ Res 2012;110:1596-603.

73. Jagannathan R, Thapa D, Nichols CE, et al. Translational Regulation of the Mitochondrial Genome Following Redistribution of Mitochondrial MicroRNA in the Diabetic Heart. Circ Cardiovasc Genet 2015;8:785-802.

74. Li H, Dai B, Fan J, et al. The Different Roles of miRNA92a-2-5p and let-7b-5p in Mitochondrial Translation in db/db Mice. Mol Ther Nucleic Acids 2019;17:424-35.

75. Place RF, Li LC, Pookot D, et al. MicroRNA-373 induces 
expression of genes with complementary promoter sequences. Proc Natl Acad Sci U S A 2008;105:1608-13.

76. Geiger J, Dalgaard LT. Interplay of mitochondrial

Cite this article as: Li H, Fan J, Chen C, Wang DW. Subcellular microRNAs in diabetic cardiomyopathy. Ann Transl Med 2020;8(23):1602. doi: 10.21037/atm-20-2205 metabolism and microRNAs. Cell Mol Life Sci 2017;74:631-46. 